ESAIM: PROCEEDINGS, May 2009, Vol. 27, p. 311-321

C. Besse, O. Goubet, T. Goudon \& S. Nicaise, Editors

\title{
SHAPE DERIVATIVE FOR A TWO-PHASE EIGENVALUE PROBLEM AND OPTIMAL CONFIGURATIONS IN A BALL ${ }^{*, * *}$
}

\author{
Carlos Conca $^{1}$, Rajesh Mahadevan ${ }^{2}$ and Leon Sanz ${ }^{3}$
}

\begin{abstract}
In this article we deal with the problem of distributing two conducting materials in a given domain, with their proportions being fixed, so as to minimize the first eigenvalue of a Dirichlet operator. When the design region is a ball, it is known that there is an optimal distribution of materials which does not involve the mixing of the materials. However, the optimal configuration even in this simple case is not known. As a step in the resolution of this problem, in this paper, we develop the shape derivative analysis for this two-phase eigenvalue problem in a general domain. We also obtain a formula for the shape derivative in the form of a boundary integral and obtain a simple expression for it in the case of a ball. We then present some numerical calculations to support our conjecture that the optimal distribution in a ball should consist in putting the material with higher conductivity in a concentric ball at the centre.
\end{abstract}

Résumé. Cet article étudie le problème de la distribution optimale de deux matériaux conducteurs aux proportions fixes, de manière à minimiser la première valeur propre d'un opérateur de Dirichlet. Dans le cas d'une boule, on sait qu'il existe une distribution optimale dans laquelle les materiaux ne se melangent pas, mais cette configuration n'est pas connue explicitement. On développe une analyse de dérivée par rapport au domaine pour ce problème spectral à double face. On fournit des arguments analytiques et numériques pour renforcer notre conjecture selon laquelle la distribution optimale dans une boule consiste à placer le matériau à conductivité plus importante dans une autre boule centrée tout au milieu. Les expériences numériques mettent en évidence ce phénomène.

\section{INTRODUCTION}

Given a bounded design region $\Omega \subset \mathbb{R}^{n}$ and two conducting materials with conductivities $\alpha$ and $\beta(0<\alpha<\beta)$, which are to be distributed in $\Omega$ so that the volume of the region $\omega$ occupied by $\beta$ is a fixed number $m$ $(0<m<|\Omega|)$, we are required to minimize the first eigenvalue of a Dirichlet problem given by

$$
\lambda^{1}(\omega):=\min _{u \in H_{0}^{1}(\Omega)} \frac{\int_{\Omega}\left(\alpha \chi_{\Omega \backslash \omega}+\beta \chi_{\omega}\right)|\nabla u|^{2} d x}{\int_{\Omega}|u|^{2} d x} .
$$

\footnotetext{
* The first author(corresponding author) thanks the MICDB for partial support through Grant ICM P05-001-F. He also thanks the Chilean 86 French Governments through Ecos-Conicyt Grant C07 E05

** The second author was supported by FONDECYT No. 1070675 and CMM, Universidad de Chile

${ }^{1}$ CMM-DIM, FCFM, Universidad de Chile, CHILE; e-mail: cconca@dim.uchile.cl

2 Departamento de Matemática, Universidad de Concepción, CHILE; e-mail: rmahadevan@udec.cl

${ }^{3}$ CMM-DIM, FCFM, Universidad de Chile, CHILE; e-mail: lsanz@dim.uchile.cl
} 
In general, given a shape design problem there may not exist a design which gives the optimal value, in which case the optimal value can only be obtained asymptotically by taking a sequence of designs which perform better and better. Typically, such sequences involve the mixing of the material phases at a microscopic scale (which goes to zero) and thus, lead to a new optimization problem where the optimization has to be done with respect to the local proportions and local geometries (the so-called micro-structure) in which the phases can be mixed. A systematic approach to these problems which involves optimizing over the microstrucutres was developed by Murat and Tartar (see [13]). In the above problem, characterizations of optimal designs involving micro-structures have been given by Cox and Lipton [3].

However, in some shape optimization problems one might be able to reach the optimum value among shapes without having to consider micro-structures. In the above problem, it has been shown in the one-dimensional case by Kren̆ [11] that, for the minimum to be attained, the material with higher conductivity needs to be distributed in the middle of the domain. In higher dimensions, when the domain is a ball, it has been shown by Alvino et. al. [2] that the infimum is attained for a radially symmetric distribution of the two materials. Recently, we have given a different proof of this result in a ball [4] using some simple ideas which have the character of being extendable to domains with partial symmetries. Surprisingly, the exact distribution of the two materials which gives the minimum value is still not known. Our conjecture, in the case of a ball, is that the infimum of the first eigenvalue, like in the one-dimensional case, is obtained by placing the stronger conductor in a concentric ball in the middle.

The problem of explicitly obtaining optimal shapes in a shape optimization problem knowing the existence of optimal shapes is a problem with its own intrinsic difficulty. A tool which is used in shape optimization problems to analyze possible local or global minimizers and to develop some algorithms for the numerical search of such minimizers is the derivative of the objective functional with respect to variations of the domain. In this article, we develop the shape derivative analysis of the first eigenvalue for the two-phase conduction problem. The main results in this respect are Theorem 1.2, where we show the existence of the shape derivative of the two-phase eigenvalue problem, and Theorem 1.4 wherein we obtain an explicit formula for it.

There is a fairly large literature on shape derivative analysis for different operators and boundary conditions cf. $[5,7,8,14-17]$. In spite of this, the shape derivative calculus for multiple-phase problems seems to have been studied very little. The shape derivatives in an inverse conductivity problem with two conducting phases was first calculated in Hettlich and Rundell [9] and later established rigorously in Afraites et. al. [1]. Our results on the shape derivative of a two-phase eigenvalue problem (see Theorem 1.2 and 1.4) seem to be the first of its kind.

The latter part of this article is dedicated to obtaining numerical evidence for our above conjecture. We plot the first eigenvalue as a function of the position of the domain containing the material having higher conductivity for certain annular and disk configurations inside a disk. The sign of the shape derivative at these configurations is also plotted. In the case of annular configurations, the formula for the shape derivative for radial perturbations simplifies to a great extent (see Proposition 2.1). Both the plots agree and indicate that it is optimal to place the material with higher conductivity in the middle.

The layout of the article is as follows. In the next section, we develop the shape derivative analysis of the first eigenvalue functional for the two-phase conduction problem through the Theorems 1.2 and 1.4. In Section 3, we state our conjecture concerning the optimal configuration in a ball and support it by some numerical results in a disk. We conclude, in the final section, proposing some future lines of work.

\section{Shape DeRIVATIVe of The First EIGENVAlue FOR TWO-PHASE CONDUCTORS}

The shape derivative is a tool which permits to understand the variation of quantities which depend on the domain (cf. Simon [14]). This is widely used in the study of shape optimization, front tracking, image segmentation problems etc. It is defined in the following way. Let us consider a functional $F$ (shape functional) which depends on the domain $\omega$. For a variation of the domain $\omega$ by a fairly smooth perturbative vector field 
$\theta$ which acts in a neighbourhood of $\omega$, the infinitesimal variation of $F$ in the direction $\theta$ is defined as

$$
F^{\prime}(\omega ; \theta)=\lim _{t \longrightarrow 0} \frac{F(\omega+t \theta)-F(\omega)}{t} .
$$

Now $F$ itself may depend on a function $u$ defined on $\omega$. So, if $u_{t}$ is the corresponding function when $\omega$ changes to $\omega_{t}:=(i d+t \theta)(\omega)$, the local derivative (also called shape derivative)and the total derivative (also called material derivative) of $u$ are defined, respectively, to be $u^{\prime}(x)=\lim _{t \rightarrow 0} \frac{u_{t}(x)-u(x)}{t}$ and $\dot{u}(x)=\lim _{t \rightarrow 0} \frac{u_{t}(x+t \theta)-u(x)}{t}$.

Remark 1.1. The shape derivative analysis in a shape optimization problem is usually quite delicate and requires careful analysis, as the perturbed quantities of interest do not depend on the perturbation field $\theta$ in an explicit way. We shall obtain these results here by a suitable application of the implicit function theorem.

We recall the setting of the spectral problem for the first eigenvalue functional before stating the existence theorem of the shape derivative. Let $\omega$ be a reference configuration with smooth boundary where the material $\beta$ is given. We take $\omega$ to be an open set whose closure is contained in $\Omega$. Given the distribution $\sigma(\omega)=\alpha \chi_{\Omega \backslash \omega}+\beta \chi_{\omega}$ of the two conductors we consider the eigenvalue problem

$$
\left\{\begin{aligned}
-\operatorname{div}(\sigma(\omega) \nabla u) & =\lambda(\omega) u \text { in } \Omega \\
u & =0 \text { on } \partial \Omega
\end{aligned}\right.
$$

Let $\lambda_{1}(\omega)$ be the first eigenvalue. It is simple and the first eigenfunction is characterized by its constant sign [12]. We normalize the first eigenfunction by assuming it to be non-negative and by taking it to satisfy

$$
\int_{\Omega}|u|^{2} d x=1
$$

We now define the admissible perturbations of $\omega$. Let $\theta$ be a smooth vector field with its support inside $\Omega$. The admissible perturbations $\omega_{t}:=\Phi_{t}(\omega)$ are the images of $\omega$ under the transformations $\Phi_{t}:=$ id $+t \theta$ for $t>0$ sufficiently small. For such $t, \Phi_{t}$ is a diffeomorphism of $\Omega$ and we have $\omega_{t} \subset \subset \Omega$. Let $\left(\lambda_{1}\left(\omega_{t}\right), u_{t}\right)$ be the normalized eigenpair corresponding to the first eigenvalue for the problem (1.2) with the configuration $\sigma\left(\omega_{t}\right)$.

Theorem 1.2. The shape derivative of the first eigenvalue $\lambda_{1}$ exists. The material derivative $\dot{u}$ of the normalized first eigenfunction $u$ exists and $\dot{u} \in H_{0}^{1}(\Omega)$. Its shape derivative $u^{\prime}$ also exists and is such that its restrictions to $\omega$ and $\Omega \backslash \bar{\omega}$ belong to $H^{1}(\omega)$ and $H^{1}(\Omega \backslash \bar{\omega})$ respectively.

Proof: We prove this result using an argument based on the Implicit Function Theorem following an established procedure which is well explained in the text [8]. The existence of the shape derivative of the first eigenvalue and the existence of the material derivative can be seen as an existence result of a smooth family of solutions for a reformulation of the perturbed eigenvalue problem. The perturbed eigenvalue problem is

$$
\left\{\begin{aligned}
-\operatorname{div}\left(\sigma\left(\omega_{t}\right) \nabla u_{t}\right) & =\lambda\left(\omega_{t}\right) u_{t} \text { in } \Omega \\
u_{t} & =0 \text { on } \partial \Omega
\end{aligned}\right.
$$

The problem (1.4) after the change of coordinates by the transformation $\Phi_{t}^{-1}$ reads

$$
\left\{\begin{aligned}
-\operatorname{div}\left(\left(\sigma\left(\omega_{t}\right) \circ \Phi_{t}\right) A_{t} \nabla\left(u_{t} \circ \Phi_{t}\right)\right) & =\lambda\left(\omega_{t}\right)\left(u_{t} \circ \Phi_{t}\right) J\left(\Phi_{t}\right) \text { in } \Omega \\
u_{t} \circ \Phi_{t} & =0 \text { on } \partial \Omega
\end{aligned}\right.
$$

where $A_{t}:=D \Phi_{t}^{-1}\left(D \Phi_{t}^{-1}\right)^{T} J\left(\Phi_{t}\right)$ and $J\left(\Phi_{t}\right)$ is the Jacobian of the transformation $\Phi_{t}$. We refer to [1,8] for the details. Thus, $\left(\lambda\left(\omega_{t}\right), u_{t}\right)$ is an eigenpair of (1.4) if and only if $\left(\lambda\left(\omega_{t}\right), u_{t} \circ \Phi_{t}\right)$ satisfies the equation (1.5). 
Furthermore, $u_{t}$ satisfies the condition $\int_{\Omega} u_{t}^{2} d x=1$ if and only if we have

$$
\int_{\Omega}\left|u_{t} \circ \Phi_{t}\right|^{2} J\left(\Phi_{t}\right) d x=1
$$

We now define $F: \mathbb{R} \times \mathbb{R} \times H_{0}^{1}(\Omega) \rightarrow H^{-1}(\Omega) \times \mathbb{R}$

$$
\begin{aligned}
F(t, \lambda, v) & :=\left(-\operatorname{div}\left(\left(\sigma\left(\omega_{t}\right) \circ \Phi_{t}\right) A_{t} \nabla v\right)-\lambda v, \int_{\Omega}|v|^{2} J\left(\Phi_{t}\right) d x-1\right) \\
& =\left(-\operatorname{div}\left(\sigma(\omega) A_{t} \nabla v\right)-\lambda v, \int_{\Omega}|v|^{2} J\left(\Phi_{t}\right) d x-1\right)
\end{aligned}
$$

in a neighbourhood of $\left(0, \lambda_{1}(\omega), u_{0}\right)$. Note that the last equality in (1.7) is due to the fact that $\sigma\left(\omega_{t}\right) \circ \Phi_{t} \equiv \sigma(\omega)$ (indeed, this is so, as $\Phi_{t}$ maps $\omega$ onto $\omega_{t}$ and the coefficient $\sigma\left(\omega_{t}\right)$ has the value $\beta$ on $\omega_{t}$ and the value $\alpha$ elsewhere on $\Omega$ while $\sigma(\omega)$ takes the values $\beta$ and $\alpha$, respectively, on the regions $\omega$ and $\Omega \backslash \omega$ ).

We now show the existence of a smooth curve of zeros passing through $\left(0, \lambda_{1}, u_{0}\right)$ for the function $F$ defined above, by verifying the hypotheses of the implicit function theorem. As $\Phi_{t}$ is a smooth function of $t$ we deduce that the maps $t \mapsto D \Phi_{t}$, and $t \mapsto A_{t}$ are smooth functions of $t$. Consequently, $F$ is a smooth function of $t$ as also in the variables $\lambda$ and $v$ being linear or quadratic in those. Now we check that $F_{\lambda, v}\left(0, \lambda_{1}(\omega), u_{0}\right): \mathbb{R} \times H_{0}^{1}(\Omega) \rightarrow H^{-1}(\Omega) \times \mathbb{R}$ is invertible. As

$$
\left\langle F_{\lambda, v}\left(0, \lambda_{1}(\omega), u_{0}\right),(\lambda, v)\right\rangle=\left(-\operatorname{div}(\sigma(\omega) \nabla v)-\lambda_{1}(\omega) v-\lambda u_{0}, 2 \int_{\Omega} v u_{0} d x\right)
$$

we now solve

$$
\begin{aligned}
-\operatorname{div}(\sigma(\omega) \nabla v)-\lambda_{1}(\omega) v-\lambda u_{0} & =f \\
2 \int_{\Omega} v u_{0} d x & =c .
\end{aligned}
$$

The first of these equations has a solution, by the spectral theory of bounded self-adjoint operators, if and only if

$$
\left\langle f+\lambda u_{0}, u_{0}\right\rangle=0 .
$$

Thus $\lambda=-\left\langle f, u_{0}\right\rangle$. Let $w$ be a particular solution of the first equation for this value of $\lambda$. The solution space is one dimensional and all the solutions are of the form $w+k u_{0}$. Plugging this in the second equation in (1.9) we have

$$
2 \int_{\Omega}\left(w+k u_{0}\right) u_{0} d x=c .
$$

This determines $k$ uniquely. Thus, the system (1.9) admits a unique solution $\left(\lambda, w+k u_{0}\right)$ which shows that the operator $F_{\lambda, v}\left(0, \lambda_{1}(\omega), u_{0}\right)$ is bijective. The continuity of the inverse follows from the Banach-Steinhaus open mapping theorem. So, by applying the Implicit Function Theorem we obtain a smooth curve of zeros $t \mapsto\left(t, \lambda_{t}, v_{t}\right)$ to $F$ in a neighbourhood of $\left(0, \lambda_{1}(\omega), u_{0}\right)$.

We now conclude the existence of the shape derivative of the first eigenvalue and the material derivative of the first eigenfuntion as follows. By our comments following the equation (1.5), $\lambda_{t}$ is an eigenvalue of the perturbed eigenvalue problem (1.4). Then we deduce that $\lambda_{t}=\lambda_{1}\left(\omega_{t}\right)$, the first eigenvalue of the perturbed problem, using the fact that the eigenvalues of a linear self-adjoint elliptic operator are discrete and vary continuously with respect to continuous perturbations of the operator [10]. Thus, as $\lambda_{t}$ is a continuous branch of eigenvalues starting at $\lambda_{1}(\omega)$, it has to coincide, locally, with the continuous branch $\lambda_{1}\left(\omega_{t}\right)$. Therefore, due to the simplicity of the first eigenfunction $u_{t}=v_{t} \circ \Phi_{t}^{-1}$ is a first eigenfunction of (1.4) and also satisfies the normalization conditions. Thus, differentiability of the branch of solutions $\left(\lambda_{t}, v_{t}\right)$ with respect to $t$ permits us to conclude, immediately, that the derivatives of $\lambda_{1}\left(\omega_{t}\right)$ and $u_{t} \circ \Phi_{t}$ exist, that is, the shape derivative of $\lambda_{1}$ and the material derivative of $u$ exist. 
Finally, the existence of the shape derivative of $u$ is a consequence of the following simple but important relation between the local and total derivatives (see Simon [14])

$$
u^{\prime}(x)=\dot{u}(x)-\theta \cdot \nabla u(x)
$$

where $u$ is the function on the unperturbed domain. On the one hand we have seen that $\dot{u} \in H_{0}^{1}(\Omega)$. On the other hand, as $\omega$ is a smooth domain and on each of $\omega$ and $\Omega \backslash \bar{\omega}, u$ satisfies an elliptic eigenvalue problem with smooth coefficients, by standard regularity theory, $u$ is smooth in each of these domains and consequently also $\nabla u$ (see Gilbarg and Trudinger [6]). As such $\nabla u$ may be not smooth across the boundary $\partial \omega$ and so, from the relation (1.10), we can only conclude that $u^{\prime} \mathrm{L}_{\omega} \in H^{1}(\omega)$ and $u^{\prime}\left\llcorner_{\Omega \backslash \bar{\omega}} \in H^{1}(\Omega \backslash \omega)\right.$.

Remark 1.3. The above theorem shows the Gateaux differentiability of the first eigenfunction $u$ in the direction of the perturbative field $\theta$. The same proof modified, while considering the deformations id $+\theta$ for sufficiently small $\theta$, will show that the first eigenfunction is Fréchet differentiable with the respect to $\theta$.

Theorem 1.4. The shape derivative of $\lambda_{1}$, given an admissible perturbation $\theta$, reads as follows

$$
\lambda_{1}^{\prime}(\omega ; \theta)=\int_{\partial \omega}\left[\sigma|\nabla u|^{2}\right] \theta \cdot n d S+2 \int_{\partial \omega}[\theta \cdot \nabla u] \sigma \nabla u \cdot n d S
$$

where $[\varphi]_{\mid \partial \omega}$ is the jump of $\varphi$ across $\partial \omega$, that is, $[\varphi]_{\mid \partial \omega}(x)=\left(\varphi_{\partial \omega^{-}}-\varphi_{\partial \omega^{+}}\right)(x)$ with $\varphi_{\partial \omega^{-}}$and $\varphi_{\partial \omega^{+}}$denoting, respectively the inner and outer trace of $\varphi$ on $\partial \omega ; n$ is the outward pointing normal (with respect to $\omega$ ) to the surface $\partial \omega$ and, $\sigma \nabla u \cdot n$ is the outward flux (with respect to $\omega$ ) at any point on $\partial \omega$.

Proof: The variational formulation of the equation (1.5) is

$$
\int_{\Omega} \sigma(\omega) A_{t}\left(\nabla u_{t} \circ \Phi_{t}\right) \cdot \nabla w d x=\int_{\Omega} \lambda\left(\omega_{t}\right)\left(u_{t} \circ \Phi_{t}\right) J\left(\Phi_{t}\right) \varphi d x \quad \text { for all } \varphi \in H_{0}^{1}(\Omega) .
$$

The integrands are continuously differentiable with respect to the variable $t$ and thus we are allowed to differentiate under the integral sign with respect to $t$ at $t=0$. Doing so, we obtain

$$
\begin{gathered}
\int_{\Omega} \sigma(\omega) \nabla \dot{u} \cdot \nabla \varphi d x+\int_{\Omega} \sigma(\omega)\left(\operatorname{div} \theta I-\left((D \theta)^{T}+D \theta\right)\right) \nabla u \cdot \nabla \varphi d x \\
=\int_{\Omega} \lambda_{1}^{\prime}(\omega ; \theta) u \varphi d x+\int_{\Omega} \lambda_{1}(\omega) \dot{u} \varphi d x+\int_{\Omega} \lambda_{1}(\omega) u \varphi \operatorname{div} \theta d x .
\end{gathered}
$$

Similarly, differentiating the relations (1.6) and the volume constraint $\left|\omega_{t}\right|=|\omega|$, written as $\int_{\omega} J\left(\Phi_{t}\right) d x=1$, with respect to $t$, we have, respectively,

$$
\begin{aligned}
& \int_{\Omega} 2 u \dot{u} d x+\int_{\Omega} u^{2} \operatorname{div} \theta d x=0 \\
& \int_{\omega} \operatorname{div} \theta d x=\int_{\partial \omega} \theta \cdot n d S=0 .
\end{aligned}
$$

Now, we shall use the above relations to deduce the expression for the shape derivative of $\lambda_{1}$. To begin with, we take $\varphi=u$ in (1.13) and use $\dot{u}$ as a test function in (1.2) to obtain

$$
\begin{aligned}
\int_{\Omega} \sigma(\omega) \nabla \dot{u} \cdot \nabla u d x+\int_{\Omega} \sigma(\omega)\left(\operatorname{div} \theta|\nabla u|^{2}-2 D \theta \nabla u \cdot \nabla u\right) d x & =\lambda_{1}^{\prime}(\omega ; \theta)+\int_{\Omega} \lambda_{1}(\omega) \dot{u} u d x+\int_{\Omega} \lambda_{1}(\omega) u^{2} \operatorname{div} \theta d x(1.1) \\
\int_{\Omega} \sigma(\omega) \nabla u \cdot \nabla \dot{u} d x & =\lambda_{1}(\omega) \int_{\Omega} u \dot{u} d x
\end{aligned}
$$


Subtracting (1.17) from (1.16) we get

$$
\int_{\Omega} \sigma(\omega)\left(\operatorname{div} \theta|\nabla u|^{2}-2 D \theta \nabla u \cdot \nabla u\right) d x=\lambda_{1}^{\prime}(\omega ; \theta)+\int_{\Omega} \lambda_{1}(\omega) u^{2} \operatorname{div} \theta d x
$$

As $\nabla u$ is smooth in each of $\omega$ and $\Omega \backslash \bar{\omega}$ we have the following identity (see [1, Theorem 3.1 equation (3.10)])

$$
\operatorname{div} \theta|\nabla u|^{2}-2 D \theta \nabla u \cdot \nabla u=-\operatorname{div}\left(2 \theta \cdot \nabla u \nabla u-|\nabla u|^{2} \theta\right)+2 \theta \cdot \nabla u \Delta u
$$

while we also have straightaway that

$$
\operatorname{div}(2 \theta \cdot \nabla u \nabla u)=2 \theta \cdot \nabla u \Delta u+2 \nabla u \cdot \nabla(\theta \cdot \nabla u) .
$$

So, from (1.19) and (1.20) we have

$$
\operatorname{div} \theta|\nabla u|^{2}-2 D \theta \nabla u \cdot \nabla u=\operatorname{div}\left(|\nabla u|^{2} \theta\right)-2 \nabla u \cdot \nabla(\theta \cdot \nabla u) .
$$

This allows us to rewrite (1.18) as

$$
\lambda_{1}^{\prime}(\omega ; \theta)=\int_{\Omega} \sigma(\omega)\left(\operatorname{div}\left(|\nabla u|^{2} \theta\right)-2 \nabla u \cdot \nabla(\theta \cdot \nabla u)\right) d x-\int_{\Omega} \lambda_{1}(\omega) u^{2} \operatorname{div} \theta d x .
$$

As both $\theta$ and $u$ are regular in each of $\omega$ and $\Omega \backslash \bar{\omega}$, from (1.2) we obtain,

$$
\begin{aligned}
\int_{\omega} \sigma(\omega) \nabla u \cdot \nabla(\theta \cdot \nabla u) d x+\int_{\partial \omega} \sigma(\omega) \nabla u \cdot n \theta \cdot \nabla u d S & =\lambda_{1}(\omega) \int_{\omega} u \theta \cdot \nabla u d x \\
& =\frac{\lambda_{1}(\omega)}{2} \int_{\omega} \theta \cdot \nabla\left(u^{2}\right) d x \\
& =-\frac{\lambda_{1}(\omega)}{2} \int_{\omega} u^{2} \operatorname{div} \theta d x+\frac{\lambda_{1}(\omega)}{2} \int_{\partial \omega} u^{2} n \cdot \theta d S .
\end{aligned}
$$

Thus, we obtain,

$$
-2 \int_{\omega} \sigma(\omega) \nabla u \cdot \nabla(\theta \cdot \nabla u) d x-\int_{\omega} \lambda_{1}(\omega) u^{2} \operatorname{div} \theta d x=2 \int_{\partial \omega} \sigma(\omega) \nabla u \cdot n \theta \cdot \nabla u d S-\lambda_{1}(\omega) \int_{\partial \omega} u^{2} n \cdot \theta d S .
$$

and, similarly,

$$
\begin{aligned}
& -2 \int_{\Omega \backslash \bar{\omega}} \sigma(\omega) \nabla u \cdot \nabla(\theta \cdot \nabla u) d x-\int_{\Omega \backslash \bar{\omega}} \lambda_{1}(\omega) u^{2} \operatorname{div} \theta d x \\
& \quad=2 \int_{\partial(\Omega \backslash \bar{\omega})} \sigma(\omega) \nabla u \cdot n \theta \cdot \nabla u d S-\lambda_{1}(\omega) \int_{\partial(\Omega \backslash \bar{\omega})} u^{2} n \cdot \theta d S .
\end{aligned}
$$

Due to the fact that $\theta$ vanishes near the boundary $\partial \Omega$, the previous equation reduces to

$$
\begin{aligned}
& -2 \int_{\Omega \backslash \bar{\omega}} \sigma(\omega) \nabla u \cdot \nabla(\theta \cdot \nabla u) d x-\int_{\Omega \backslash \bar{\omega}} \lambda_{1}(\omega) u^{2} \operatorname{div} \theta d x \\
& \quad=2 \int_{\partial \omega}(\sigma(\omega) \nabla u \cdot n)_{\partial \omega, \Omega \backslash \bar{\omega}}(\theta \cdot \nabla u)_{\partial \omega^{+}} d S-\lambda_{1}(\omega) \int_{\partial \omega} u_{\partial \omega^{+}}^{2} n_{\partial \omega^{+}} \cdot \theta d S,
\end{aligned}
$$


where $(\sigma(\omega) \nabla u \cdot n)_{\partial \omega, \Omega \backslash \omega^{\prime}}$ is the outward flux for the domain $\Omega \backslash \bar{\omega},(\cdot)_{\partial \omega^{+}}$the trace on $\partial \omega$ with respect to $\Omega \backslash \bar{\omega}$ and $n_{\partial \omega^{+}}$is the outward normal on $\partial \omega$ with respect to $\Omega \backslash \bar{\omega}$. We remark that the exterior normals to $\partial \omega$ with respect to $\omega$ and $\Omega \backslash \bar{\omega}$ are the negatives of one another, we have the transmission condition $(\sigma \nabla u \cdot n)_{\partial \omega, \omega}=-(\sigma \nabla u \cdot n)_{\partial \omega, \Omega \backslash \bar{\omega}}$ and as $u$ belongs to $H_{0}^{1}(\Omega)$ its trace on the smooth interface $\partial \omega$ is unique, so that $u_{\partial \omega^{+}}=u_{\partial \omega^{-}}$. So, by adding the equations (1.23) and (1.24) we obtain

$$
-2 \int_{\Omega} \sigma(\omega) \nabla u \cdot \nabla(\theta \cdot \nabla u) d x-\int_{\Omega} \lambda_{1}(\omega) u^{2} \operatorname{div} \theta d x=2 \int_{\partial \omega} \sigma(\omega) \nabla u \cdot n[\theta \cdot \nabla u] d S .
$$

Also observe that

$$
\begin{aligned}
\int_{\Omega} \sigma(\omega) \operatorname{div}\left(|\nabla u|^{2} \theta\right) d x & =\int_{\omega} \sigma(\omega) \operatorname{div}\left(|\nabla u|^{2} \theta\right) d x+\int_{\Omega \backslash \bar{\omega}} \sigma(\omega) \operatorname{div}\left(|\nabla u|^{2} \theta\right) d x \\
& =\int_{\partial \omega} \sigma(\omega)|\nabla u|^{2} \theta \cdot n d S+\int_{\partial \omega}\left(\sigma(\omega)|\nabla u|^{2}\right)_{\partial \omega^{+}} \theta \cdot n_{\partial \omega^{+}} d S \\
& =\int_{\partial \omega}\left[\sigma(\omega)|\nabla u|^{2}\right] \theta \cdot n d S .
\end{aligned}
$$

So, we conclude (1.11) using (1.22), (1.25) and (1.26).

\section{Minimizing DistribUtion IN A DISK}

We know by the results of [2] and [4] that there exists minimizing configurations in a ball which are radially symmetric. This means that the materials are to be distributed in various spherical shells. We have the following conjecture.

Conjecture: For the problem of distributing fixed quantities of two conducting materials in a disk so as to minimize the first eigenvalue, the infimum value is attained when the material with higher conductivity is placed in a concentric disk in the middle.

We now give evidence to our conjecture in a disk by plotting numerically the eigenvalues and the shape derivative of the eigenvalues given by formula (1.11) for some annular configurations in a disk. We first simplify the formula for the shape derivative for radial perturbations of radially symmetric initial configurations.

Proposition 2.1. Let's assume that the two materials are distributed in a finite number of concentric annuli. Whenever there is a layer of $\beta$ included between two layers of $\alpha, \lambda_{1}^{\prime}(\omega ; \theta)$ for a radially symmetric perturbation $\theta$ of $\omega$ conserving the volumes of $\alpha$ and $\beta$ reads

$$
\lambda_{1}^{\prime}\left(\omega_{0} ; \theta\right)=3\left(\frac{1}{\beta}-\frac{1}{\alpha}\right)\left\{\left((\sigma|\nabla u|)_{S_{1}}\right)^{2}-\left((\sigma|\nabla u|)_{S_{2}}\right)^{2}\right\} \theta \cdot n_{S_{1}} \operatorname{per}\left(S_{1}\right) .
$$

Here, $\operatorname{per}\left(S_{1}\right)$ stands for the perimeter of $S_{1}$.

Proof: Let the conductivity coefficient in the reference configuration be $\sigma$. This is a radial function. Let $u$ be a normalized first eigenfunction for the conductivity problem in the reference configuration. Let us concentrate on a layer $\omega_{0}$ of $\beta$ included between two layers of $\alpha$ and let us write its boundary as $S_{1} \cup S_{2}$ where $S_{1}$ and $S_{2}$ are, respectively, the inner and outer boundaries. We consider a radially symmetric perturbation $\theta$ whose support is contained within a neighbourhood of $\omega_{0}$ in $\Omega$ and is such that it conserves the volume of $\omega_{0}$. The formula (1.11) for the shape derivative $\lambda_{1}^{\prime}\left(\omega_{0} ; \theta\right)$ reads as follows:

$$
\begin{aligned}
\lambda_{1}^{\prime}\left(\omega_{0} ; \theta\right)= & \int_{S_{1}}\left[\sigma|\nabla u|^{2}\right] \theta \cdot n d S+\int_{S_{2}}\left[\sigma|\nabla u|^{2}\right] \theta \cdot n d S \\
& +2 \int_{S_{1}}[\theta \cdot \nabla u] \sigma \nabla u \cdot n d S+2 \int_{S_{2}}[\theta \cdot \nabla u] \sigma \nabla u \cdot n d S,
\end{aligned}
$$


where $n$ is the outward pointing normal, with respect to the band $\omega_{0}$, at any of the surfaces $S_{1}, S_{2}$.

To begin with, as $\sigma$ is radially symmetric it can be shown that the eigenfunction $u$ is radially symmetric. So, as $\theta, n$ are also radially symmetric functions, the integrands in all the boundary integrals are radially symmetric and hence constant on the surfaces $S_{1}$ and $S_{2}$. Thus, we may write

$$
\begin{aligned}
\lambda_{1}^{\prime}\left(\omega_{0} ; \theta\right)= & {\left[\sigma|\nabla u|^{2}\right]_{\left.\right|_{S_{1}}} \theta \cdot n_{S_{1}} \operatorname{per}\left(S_{1}\right)+\left[\sigma|\nabla u|^{2}\right]_{\left.\right|_{S_{2}}} \theta \cdot n_{S_{2}} \operatorname{per}\left(S_{2}\right) } \\
& +2[\theta \cdot \nabla u]_{S_{S_{1}}}(\sigma \nabla u)_{S_{1}^{-}} \cdot n_{S_{1}} \operatorname{per}\left(S_{1}\right)+2[\theta \cdot \nabla u]_{S_{S_{2}}}(\sigma \nabla u)_{S_{2}^{-}} \cdot n_{S_{2}} \operatorname{per}\left(S_{2}\right)
\end{aligned}
$$

where $(\cdot)_{S_{i}^{-}}, i=1,2$, denotes the inner trace on $S_{i}$, with respect to $\omega_{0}$.

Now, we try to obtain simplifications of the above formula. To reduce the expression in the second line of (2.2) we first deduce that, for $i=1,2$, we have

$$
[\theta \cdot \nabla u]_{\left.\right|_{S_{i}}}(\sigma \nabla u)_{S_{i}^{-}} \cdot n_{S_{i}} \operatorname{per}\left(S_{i}\right)=\left[\sigma|\nabla u|^{2}\right]_{\left.\right|_{S_{i}}} \theta \cdot n_{S_{i}} \operatorname{per}\left(S_{i}\right) .
$$

Indeed, using $\nabla u=\nabla u \cdot n n$ in $\theta \cdot \nabla u, u$ being a radial function, we have

$$
\begin{aligned}
{[\theta \cdot \nabla u]_{S_{S_{i}}}(\sigma \nabla u)_{S_{i}^{-}} \cdot n_{S_{i}} \operatorname{per}\left(S_{i}\right) } & =\left(\nabla u_{S_{i}^{-}} \cdot n_{S_{i}}-\nabla u_{S_{i}^{+}} \cdot n_{S_{i}}\right)(\sigma \nabla u)_{S_{i}^{-}} \cdot n_{S_{i}} \theta \cdot n_{S_{i}} \operatorname{per}\left(S_{i}\right) \\
& =\left\{\sigma_{S_{i}^{-}}\left(\nabla u_{S_{i}^{-}} \cdot n_{S_{i}}\right)^{2}-\sigma_{S_{i}^{+}}\left(\nabla u_{S_{i}^{+}} \cdot n_{S_{i}}\right)^{2}\right\} \theta \cdot n_{S_{i}} \operatorname{per}\left(S_{i}\right) \\
& =\left[\sigma|\nabla u|^{2}\right]_{S_{S_{i}}} \theta \cdot n_{S_{i}} \operatorname{per}\left(S_{i}\right)
\end{aligned}
$$

where the penultimate equality is obtained applying the transmission condition $\sigma_{S_{i}^{-}} \nabla u_{S_{i}^{-}} \cdot n_{S_{i}}=\sigma_{S_{i}^{+}} \nabla u_{S_{i}^{+}} \cdot n_{S_{i}}$ and the last equality follows observing that $|\nabla u|_{S_{i}^{ \pm}}=\left|\nabla u_{S_{i}^{ \pm}} \cdot n_{S_{i}}\right|$. Furthermore, these relations imply that $\sigma_{S_{i}^{-}}|\nabla u|_{S_{i}^{-}}=\sigma_{S_{i}^{+}}|\nabla u|_{S_{i}^{+}}, i=1,2$ which allows us to obtain

$$
\left[\sigma|\nabla u|^{2}\right]_{S_{i}}=\left(\frac{1}{\beta}-\frac{1}{\alpha}\right)\left((\sigma|\nabla u|)_{S_{i}}\right)^{2}
$$

By the above observation and the relations (2.3)-(2.4) and the conservation of mass condition (1.15) which yields $\theta \cdot n_{S_{1}} \operatorname{per}\left(S_{1}\right)+\theta \cdot n_{S_{2}} \operatorname{per}\left(S_{2}\right)=0$, the formula $(2.2)$ reduces finally to

$$
\lambda_{1}^{\prime}\left(\omega_{0} ; \theta\right)=3\left(\frac{1}{\beta}-\frac{1}{\alpha}\right)\left\{\left((\sigma|\nabla u|)_{S_{1}}\right)^{2}-\left((\sigma|\nabla u|)_{S_{2}}\right)^{2}\right\} \theta \cdot n_{S_{1}} \operatorname{per}\left(S_{1}\right)
$$

Remark 2.2. It should be interesting to test, using this formula, whether the first eigenvalue can be lowered by perturbing an annulus containing the material $\beta$ towards the centre. This requires comparing the values of $(\sigma|\nabla u|)^{2}$ on the two edges of the annular region and could probably be achieved using the methods of partial differential equations.

Numerical Results: The numerical results which are to be presented now were obtained using FREEFEM. Essentially, we calculate the eigenvalues for certain configurations of our interest and also calculate the shape derivative at these configurations for certain perturbations using the contour integral (1.11) or the simplified formula (2.1). To make these calculations the domain for a given configuration is meshed using FREEFEM by specifying the number of boundary elements. The eigenvalues are then calculated in a standard way from the variational formulation. While calculating the contour integral (1.11) which gives the shape derivative we have 
used the library function jump of FREEFEM. The only information about the shape derivative which will be of significance to our experiments will be its sign.

In the first of these experiments, we consider a domain which is a disk of unit radius and we assume the material $\beta$ to be placed in an annular region having internal and external radius $r_{1}$ and $r_{2}$ respectively within a disk and the material $\alpha$, in the complement of this annulus within the unit disk. Let $m$ be the proportion of the total volume that the material $\beta$ occupies so that $m=\left(r_{2}^{2}-r_{1}^{2}\right)$. We take $\theta$ to be the radial perturbation which moves the annulus containing $\beta$ away from the centre while maintaining its area fixed.

In Figure 1 we plot the calculated numerical values of the first eigenvalue and the shape derivative as a function of the internal radius for the values $\alpha=1, \beta=2$ and the proportions $m=0.1,0.5$, and 0.9 respectively. The shape derivative, in this case, is calculated using the simpler formula (2.1) after writing the terms $(\sigma|\nabla u|)_{S_{i}}^{2} \theta \cdot n_{S_{i}} \operatorname{per}\left(S_{i}\right)$ as surface integrals $\int_{S_{i}}(\sigma|\nabla u|)_{S_{i}}^{2} \theta \cdot n_{S_{i}} d S$. We do the same for $\alpha=1, \beta=200$ in Figure 2.
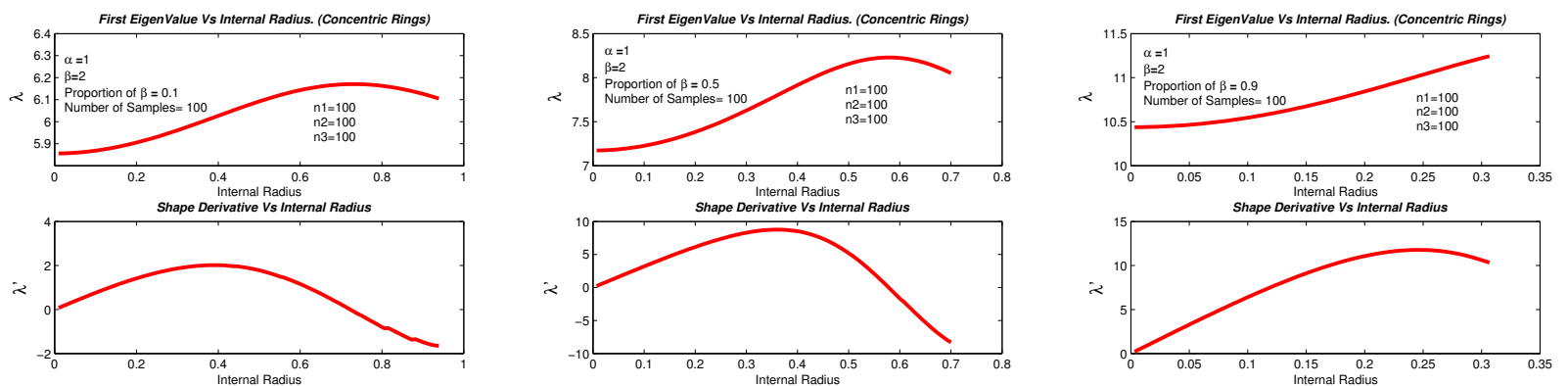

Figure 1. Concentric disks for $\beta=2$
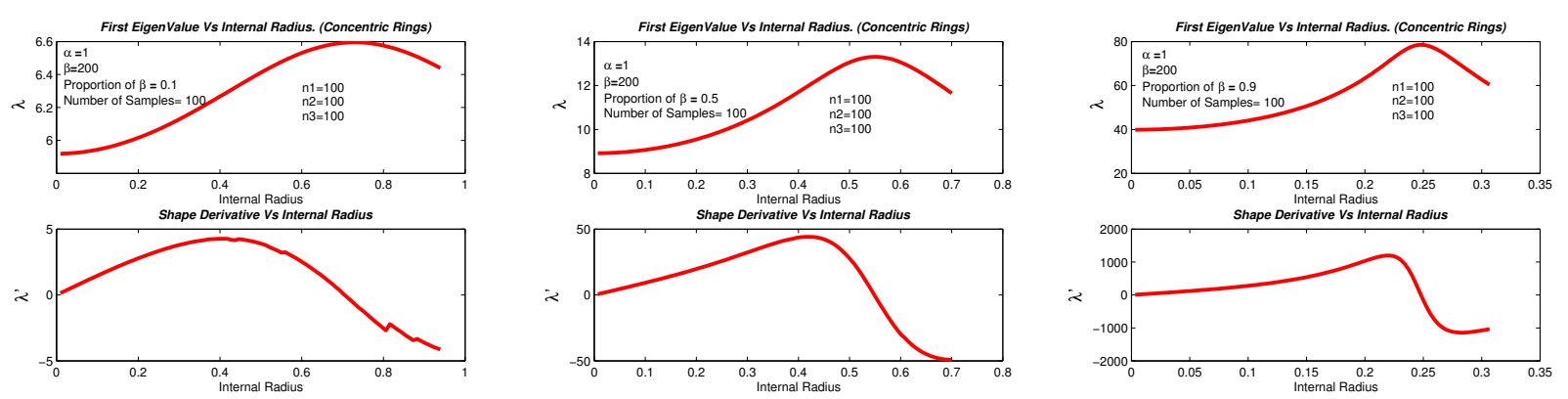

FiguRE 2. Concentric disks for $\beta=200$

The numbers $n_{1}, n_{2}$ and $n_{3}$ refer to the number of boundary nodes on the circles with radius $r_{1}, r_{2}$ and 1 respectively.

We make the following observations.

In all the cases we see that the first eigenvalue is the smallest for $r_{1}=0$ which corresponds to taking the material $\beta$ in the middle. The sign of the calculated shape derivative is positive in the intervals where $\lambda_{1}$ is seen to be increasing with respect to $r_{1}$ and is negative where $\lambda_{1}$ is seen to decrease with $r_{1}$. As we know that the positive sign of the shape derivative means that the perturbation $\theta$ increases the value of the eigenvalue functional and viceversa, we observe that the graph of the shape derivatives reflects the behaviour seen in the graph of the first eigenvalue function. 
Although, we are guaranteed by the theorem of Alvino et. al. [2] that the minimization problem admits a radially symmetric minimizer it does not exclude the presence of other minimizers which do not have radial symmetry. With this in mind, we consider an experiment in a unit disk where the material $\beta$ is put in a smaller disk whose area is a fraction $m$ of the total area. The perturbation $\theta$ is the translation of the smaller disk outwards along the radial direction.

In Figure 3 we plot the first eigenvalue and the shape derivative as a function of the distance $l$ between the centers of the two non-concentric disks taking $\alpha=1, \beta=2$ and for the proportions $m=0.1,0.5$, and 0.9 . We do the same for $\alpha=1, \beta=200$ in Figure 4 .
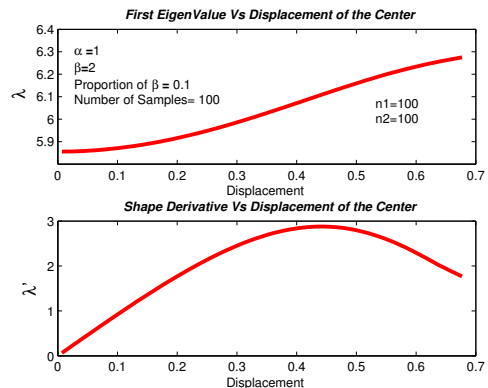
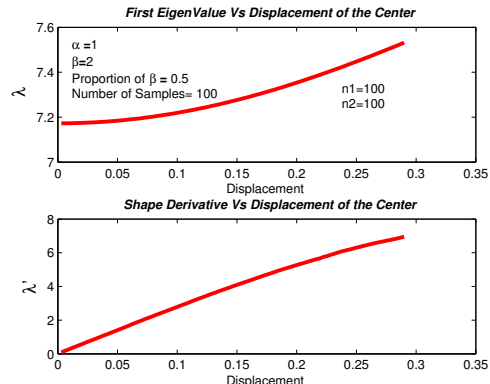
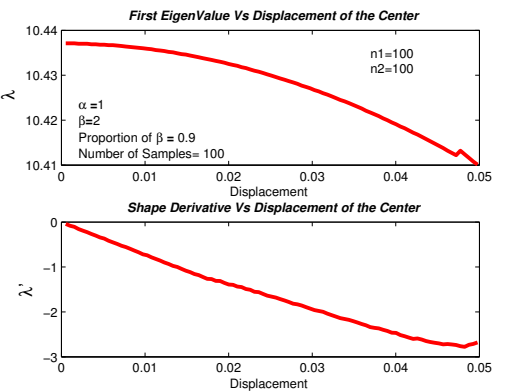

Figure 3. Non-concentric disks configurations for $\beta=2$

The numbers $n_{1}$ and $n_{2}$ refer to the number of nodes on the boundaries of the inner and outer disks, respectively.

In general we see that we obtain smaller values for the eigenvalue when the inner disk is near the centre of the domain. Also, in general, we see that the sign of the shape derivative agrees with the behaviour shown the eigenvalue graph.

However, there are some cases where these do not seem to be the case. More precisely, we see when we take $\alpha=1$ and $\beta=2$ and $m=0.9$ that the eigenvalue seems to be smaller when the disk is displaced. Actually, in carrying out the experiment for values of $\beta$ of nearly the same order as $\alpha$ (for values not bigger than 2) a strange behaviour seems to arise as the proportion $m$ approaches the value 0.755 and onwards. We try to explain this unexpected behaviour in the following way. One of the things to consider here is that the variation of the eigenvalues is of the order of $10^{-3}$ or less. In addition, the condition numbers of the matrices involved are very high (of the order $10^{28}$ ) due to the Dirichlet condition, and it becomes hard to distinguish the true variations in the calculated eigenvalues from the variation due to the numerical errors.
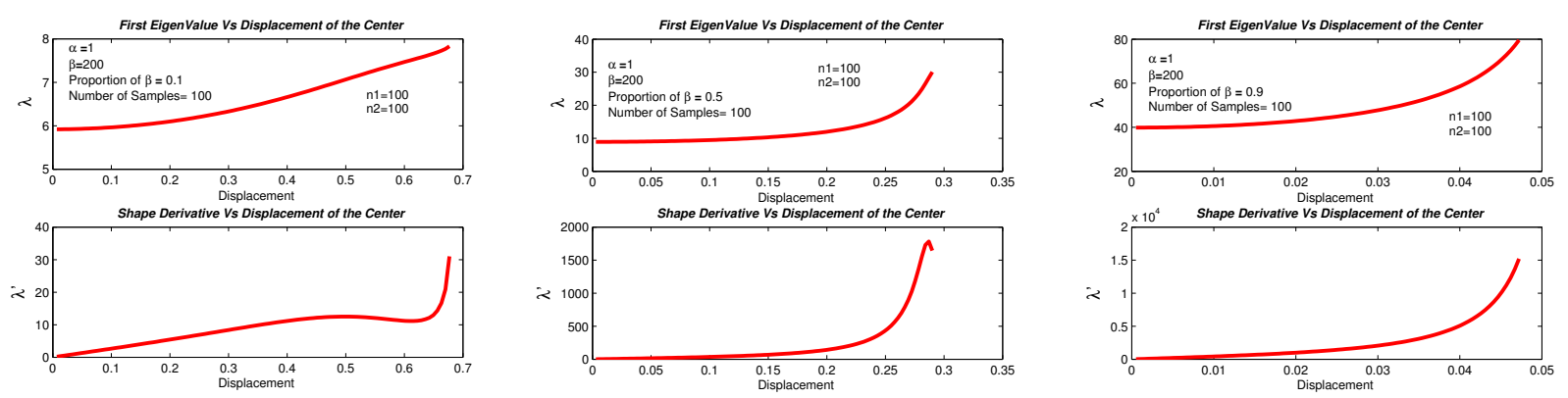

Figure 4. Non-concentric disks configurations for $\beta=200$ 
We also see that the numerical values of shape derivative calculated using formula (1.11) behaves chaotically and show little correlation with the variations of the eigenvalue. Certainly we are inside a turbulent numerical region.

The numerical results were observed to normalize for values of the coefficient $\beta$ from 2.1 onwards.

\section{Conclusions}

On the basis of the above numerical results we reach the conclusion that in domains like disks it may be better to place the material with higher conductivity in the middle in order to minimize the first eigenvalue. The shape derivative has been used in a limited way in this article to see the effect of some specific perturbations on certain configurations. It would be interesting to exploit the shape derivative calculated in this article in looking for directions of descent and in the development of algorithms which could help in automatic detection of the optimal distribution.

Acknowledgements: We would like to thank the referees of the article whose critical comments and helpful suggestions, in the form of pertinent references, have been important for a rigorous presentation of the results on shape derivative and for the rectification of some crucial errors. We would also like to thank Patricio Cumsille for useful discussions on the numerical computations involved.

\section{REFERENCES}

[1] Afraites L, Dambrine M, Kateb D (2007) Shape methods for the transmission problem with a single measurement. Numerical Functional Analysis and Optimization 28(5) 519-551.

[2] Alvino A, Lions PL, Trombetti G (1989) Optimization problems with prescribed rearrangements. Nonlinear Analysis TMA 13(2): 185-220.

[3] Cox S, Lipton R (1996) Extremal eigenvalue problems for two-phase conductors. Arch. Rational Mech. Anal. 136: $101-117$.

[4] Conca C, Mahadevan R, Sanz L (2008) An extremal eigenvalue problem for a two-phase conductor in a ball, published online in Appl. Math. Optim.

[5] Fernández Bonder JF, Groisman P, Rossi JD (2007), Optimization of the first Steklov eigenvalue in domains with holes: a shape derivative approach. Ann. Mat. Pura. Appl. 186(2), 341-358.

[6] Gilbarg, Trudinger, Elliptic partial differential equations of second order, Springer.

[7] Henrot A (2006) Extremum Problems for Eigenvalues of Elliptic Operators. Birkhaüser.

[8] Henrot A, Pierre M (2005) Variation et Optimisation de Formes. Mathématiques et Applications 48. Springer.

[9] Hettlich F, Rundell W (1998) The determination of a discontinuity from a single boundary measurement. Inverse Problems $14,67-82$.

[10] Kato T (1966) Perturbation Theory for Linear Operators, Classics in Mathematics. Springer-Verlag.

[11] Kreı̆ MG (1955) On certain problems on the maximum and minimum of characteristic values and on the Lyapunov zones of stability. AMS Translations Ser. 2(1): 163-187.

[12] Krĕn MG, Rutman MA (1950) Linear operators leaving invariant a cone in a Banach space. Amer. Math. Soc. Translation no. 26 .

[13] Murat F, Tartar L (1997) Calculus of Variations and Homogenization (engl. transl. of original french article) in "Topics in the Mathematical Modelling of Composite materials" Eds. A. Cherkaev and R.V. Kohn, PNLDE 31. Birkhaäuser.

[14] Simon J (1980) Differentiation with respect to the domain in boundary value problems. Numer. Funct. and Optimiz. 2(7-8), 649-687.

[15] Schiffer M (1946) Hadamard's formula and variations of domain functions. Amer. J. Math. 68, 417-448.

[16] Shimakura N (1983) La première valeur propre du Laplacien pour le problème de Dirichlet. J. Math. Pures. Appl. 62(2), 129-152.

[17] Sokolowski J, Zolesio JP (1992) Introduction to Shape Optimization: Shape Sensitivity Analysis. Springer Series in Computational Mathematics 10. Springer. Berlin. 